\title{
Elucidating lactate oxidase enzyme-biomarker lactate interactions by SAXS, crystallography and X-ray footprinting for developing a real-time stress biosensor.
}

\author{
Rohit Jain ${ }^{1}$, James Byrnes ${ }^{2}$, David Chang ${ }^{3}$, Erik R Farquhar ${ }^{4}$, David T Lodowski ${ }^{5}$, Mark R Chance ${ }^{6}$ \\ ${ }^{1}$ No affiliation given ${ }^{2}$ Brookhaven National Lab, ${ }^{3}$ Case Western Reserve University, ${ }^{4}$ Brookhaven \\ National Lab, ${ }^{5}$ Case Western Reserve University, ${ }^{6}$ Case Western Reserve University \\ rohit.mpibpc@gmail.com
}

\begin{abstract}
Wearable biosensors offer unique possibilities for in situ, real-time, and non-invasive monitoring of health and performance. Immobilized bacterial lactate oxidase enzyme (LOx) is a key biosensor candidate for sensing the excreted biomarker lactate in biofluids such as eccrine sweat. A readout of LOx sites involved in substrate binding will help to adjust the enzymatic activity and specificity for engineering LOx as a wearable biosensor. In this direction, we expressed and purified recombinant LOx from Aerococcus viridans as a candidate recognition module for the hypoxic biomarker, lactate. After several rounds of crystallization, X-ray data collection, model building and refinement, we determined the crystal structure of LOx at $1.17 \AA$, the highest resolution determined to date. SAXS studies corroborated the crystallographic tetramer as native and solution state structure. No change in oligomeric state nor were large scale changes in structure observed upon binding lactate. We then employed X-ray footprinting mass spectrometry (XFP-MS), an in situ hydroxyl radical (OH) labeling method, which covalently modifies amino acid side chains which are solvent accessible to probe changes in protein dynamics/solvent accessibility. XFP-MS revealed conformational changes in LOx in the solution state in response to lactate binding. Different LOx segments show protection and deprotection in LA bound state. More specifically, changes in LOx solvent accessibility were observed in regions observed in the crystal structure to have high B factors and more static disorder, specifically in a loop that caps the FMN cofactor and lactate substrate site. Combined, these studies highlight the complimentary use of multiple structural biophysical techniques for elucidating molecular level interactions in a key metabolic and biomedically important enzyme. Moving forward, above structural studies provided a platform to tune LOx structure for optimizing its use as a real-time stress biosensor.
\end{abstract}

